BULLETIN OF THE

AMERICAN MATHEMATICAL SOCIETY

Volume 77, Number 6, November 1971

\title{
THE CARDINALITY OF FIRST COUNTABLE SPACES
}

\author{
BY PRABIR ROY 1
}

Communicated by Steve Armentrout, March 19, 1971

ABstract. Arhangelskil has obtained, as a consequence of a general theorem of his, that if a compact Hausdorff space $X$ satisfies the first countability axiom then $|X| \leqq c\left(=2 \aleph_{0}\right)$. What is perhaps a somewhat simpler proof of above is offered here.

Recently Arhangelskii has shown [1] that if a compact Hausdorff space $X$ satisfies the first countability axiom then $|X| \leqq c\left(=2^{N_{0}}\right)$. This may be proved by constructing a nonincreasing net $Y$ of closed sets covering $X$ where the domain of the net $Y$ is the collection $K$ of graphs of nonnegative real valued functions (henceforth to be called sequences) whose domains are the proper initial segments of the naturally well-ordered set of countable ordinals, with $K$ partially ordered by set inclusion. Note that $K$ with such a partial ordering is a tree of width $c$ and height $\boldsymbol{\aleph}_{1}$. The details are as follows.

I. If a Hausdorff space is Lindelöf on a closed subset $L$ with $|L| \leqq c$ and the first countably axiom holds in $X$ at each point of $L$, then $L$ is the intersection of $c$ (or less) open sets. To see this, choose a countable basis for each point of $L$. This gives a collection $J$ of open sets with $|J| \leqq c$. Let $H$ denote the collection of all the unions of countable subcollections of $J$ covering $L .|H| \leqq c$, and in the presence of Hausdorff and Lindelöf, the common part of all the members of $H$ is $L$.

II. Let $Y(0)$ be a closed set such that $0<|Y(0)| \leqq c$. Since by I, $Y(0)$ is the intersection of $c$ (or less) open sets, $X-Y(0)$ is the union of $c$ closed subsets $Y(r)$ ( $r$ positive real number) such that $Y(0)$ $\cap Y(r)=\varnothing$. These sets need not be mutually disjoint, distinct, or for that matter nonvoid but in visualizing the net it may simplify thinking about the inclusion properties to picture them as mutually disjoint. Now if $r_{1}$ and $r_{2}$ are arbitrary nonnegative real numbers, $Y\left(r_{1}, r_{2}\right)$ is a closed subset of $Y\left(r_{1}\right)$ with the following properties:

(a) If $\left|Y\left(r_{1}\right)\right| \leqq c, Y\left(r_{1}, r_{2}\right)=\varnothing$, but

(b) if $\left|Y\left(r_{1}\right)\right|>c$, then $0<\left|Y\left(r_{1}, 0\right)\right| \leqq c$ and, for $r_{2}>0, Y\left(r_{1}, 0\right)$ $\cap Y\left(r_{1}, r_{2}\right)=\varnothing$ and

$A M S 1970$ subject classifications. Primary 54A25, 54D20; Secondary 04X00, 04A10.

Key words and phrases. First countability axiom, compact, Hausdorff, cardinality of the continuum, trees.

${ }^{1}$ Partially supported by NSF GP 11699 and ghost written by F. B. Jones.

Copyright (c) American Mathematical Society 1971 
(c) $\mathrm{U}_{r_{2}>0} Y\left(r_{1}, r_{2}\right)=Y\left(r_{1}\right)-Y\left(r_{1}, 0\right)$.

In this same manner each $Y\left(r_{1}, r_{2}, r_{3}\right)$ is defined, and in general $Y\left(r_{1}, r_{2}, \cdots, r_{z}, \cdots\right)(z<\alpha)$ is defined when $\alpha(\alpha<\Omega)$ is a nonlimit ordinal and also $\alpha-1$, the immediate predecessor of $\alpha$, is a nonlimit ordinal.

III. Now suppose that $r_{1}, r_{2}, \ldots$ is a $\omega$ sequence of nonnegative reals such that for each natural number $n, Y\left(r_{1}, r_{2}, \cdots, r_{n}\right)$ has been defined. Of course each such set is closed and $Y\left(r_{1}\right) \supset Y\left(r_{1}, r_{2}\right)$ $\supset Y\left(r_{1}, r_{2}, r_{3}\right) \supset \ldots$. Let

$$
Y\left(r_{1}, r_{2}, \cdots\right)=Y\left(r_{1}\right) \cap Y\left(r_{1}, r_{2}\right) \cap Y\left(r_{1}, r_{2}, r_{3}\right) \cap \cdots .
$$

In this manner $Y\left(r_{1}, r_{2}, \cdots, r_{z}, \cdots\right)(z<\alpha)$ is defined for $\alpha$ a countable limit ordinal.

IV. As before if $\left|Y\left(r_{1}, r_{2}, \cdots\right)\right| \leqq c$ then $Y\left(r_{1}, r_{2}, \cdots, r_{\omega}\right)=\varnothing$ $\left(0 \leqq r_{\omega}\right)$. But if $\left|Y\left(r_{1}, r_{2}, \cdots\right)\right|>c$, then it is the union of the closed subsets $Y\left(r_{1}, r_{2}, \cdots, r_{\omega}\right)\left(0 \leqq r_{\omega}\right)$ such that

(1) $Y\left(r_{1}, r_{2}, \cdots, 0\right) \cap Y\left(r_{1}, r_{2}, \cdots, r_{\omega}\right)=\varnothing$ for $r_{\omega}>0$,

(2) $0<\left|Y\left(r_{1}, r_{2}, \cdots, 0\right)\right| \leqq c$ and

(3) $Y\left(r_{1}, r_{2}, \cdots, 0\right)$ contains all those limit points of $D=Y(0)$ $\cup Y\left(r_{1}, 0\right) \cup Y\left(r_{1}, r_{2}, 0\right) \cup Y\left(r_{1}, r_{2}, r_{3}, 0\right) \cup \cdots$ that belong to $Y\left(r_{1}, r_{2}, \cdots\right)$.

This condition (3) is very important in continuing the definition at a branching level which is the limit of branching levels. But since $X$ is first countable, $|\bar{E}| \leqq c$ for any subset $E$ with $|E| \leqq c$. Hence $|\bar{D}| \leqq c$ and $\left|\bar{D} \cap Y\left(r_{1}, r_{2}, \cdots\right)\right| \leqq c$. So the existence of $Y\left(r_{1}, r_{2}, \cdots, 0\right)$ is evident. Actually $\bar{D}-D \subset Y\left(r_{1}, r_{2}, \cdots\right)$ but we do not need that fact right here. Once $Y\left(r_{1}, r_{2}, \cdots, 0\right)$ is defined, the validity of the definition of $Y\left(r_{1}, r_{2}, \cdots, r_{\omega}\right)\left(r_{\omega}>0\right)$ follows from I. In this manner $Y\left(r_{1}, r_{2}, \cdots, r_{z}, \cdots\right)(z<\alpha)$ is defined when $\alpha$ is a countable nonlimit ordinal but $\alpha-1$ is a limit ordinal.

Without going through the formal inductive construction, the reader can see how $Y$ is defined over all of the tree $K$.

Now suppose that over some branch of $K$ with height equal to $\boldsymbol{N}_{1}$, $Y$ fails to take on the value $\varnothing$ from some level on. That is, there is an uncountable sequence $r_{1}, r_{2}, \cdots, r_{z}, \cdots(z<\Omega)$ of nonnegative reals such that for each $\alpha<\Omega,\left|Y\left(r_{1}, r_{2}, \cdots, r_{z}, \cdots\right)(z<\alpha)\right|>c$. Note that then it must be that $r_{z}>0$ for each $z<\Omega$. But

$$
\begin{gathered}
E=Y(0) \cup Y\left(r_{1}, 0\right) \cup Y\left(r_{1}, r_{2}, 0\right) \cup \\
\cdots \cup Y\left(r_{1}, r_{2}, \cdots, r_{z}, \cdots, 0\right)(z<\alpha) \cup \cdots \quad(\alpha<\Omega)
\end{gathered}
$$

is a closed set. To see this, suppose on the contrary that $p \in \bar{E}-E$. By using successively the first countability of $X$, the closedness of 
$Y\left(r_{1}, r_{2}, \cdots, r_{2}, \cdots, 0\right)(z<\alpha)$ for each $\alpha<\Omega$, and the induced wellordering of the collection $Y(0), Y\left(r_{1}, 0\right), \cdots$, one obtains a $\omega$ sequence of points $q_{1}, q_{2}, \cdots$ converging to $p$ and an increasing $\omega$ sequence of countable ordinals $1<\alpha_{1}<\alpha_{2}<\alpha_{3}<\cdots$ such that for each positive integer $i$,

$$
q_{i} \in Y\left(r_{1}, r_{2}, \cdots, r_{3}, \cdots, 0\right) \quad\left(z<\alpha_{i}\right) .
$$

Let $\beta=$ l.u.b. $\left\{\alpha_{i}\right\}_{i=1}^{\infty}<\Omega$. Then $\beta$ is a limit ordinal and, by IV, we have that $p \in Y\left(r_{1}, r_{2}, \cdots, r_{z}, \cdots, 0\right)(z<\beta)$, namely, a contradiction. So, $E$ is closed. Also $|E| \leqq c$. But, $X-Y\left(r_{1}\right), X-Y\left(r_{1}, r_{2}\right)$, $X-Y\left(r_{1}, r_{2}, r_{3}\right), \cdots, X-Y\left(r_{1}, r_{2}, \cdots, r_{z}, \cdots\right)(z<\alpha), \cdots$ $(\alpha<\Omega)$ is a collection of open sets covering $E$ which contains no countable covering subcollection. This is impossible in a compact space or a Lindelöf space.

This means that for each point $x \in X$ there is at least one (possibly more than one) ordinal $\alpha(\alpha<\Omega)$ and a sequence $r_{1}, r_{2}, \cdots$, $r_{z}, \cdots(z<\alpha)$ such that $x \in Y\left(r_{1}, r_{2}, \cdots, r_{z}, \cdots\right)$ and $\left|Y\left(r_{1}, r_{2}, \cdots, r_{z}, \cdots\right)\right| \leqq c$. Since there are no more than $c$ nonvoid sets in the entire image of the net $Y,|X| \leqq c$. So we have established the following theorem:

THEOREM. If a Hausdorff space $X$ is Lindelöf on every closed subset $L$ when $|L| \leqq c$ and the first countability axiom holds true in $X$, then $|X| \leqq c$.

Comment of the Writer. The above argument appears to be less involved than Arhangelskil's, due partly to the fact that his results are more general. Quite clearly Roy's argument may be generalized. In another connection the writer has done a generalization of this sort [2]. A kind of converse appears in [3].

\section{REFERENCES}

1. A. V. ArhangelskiY, On the cardinality of bicompacta satisfying the first axiom of countability, Dokl. Akad. Nauk SSSR 187 (1969), 967-970=Soviet Math. Dokl. 10 (1969), 951-955. MR 40 \#4922.

2. F. B. Jones, Fake Souslin trees, Duke Math. J. 36 (1969), 571-574.

3. - On the first countability axiom for locally compact Hausdorff spaces, Colloq. Math. 7 (1959), 33-34. MR 22 \#2972.

State University of New York, Binghamton, New York 13901 